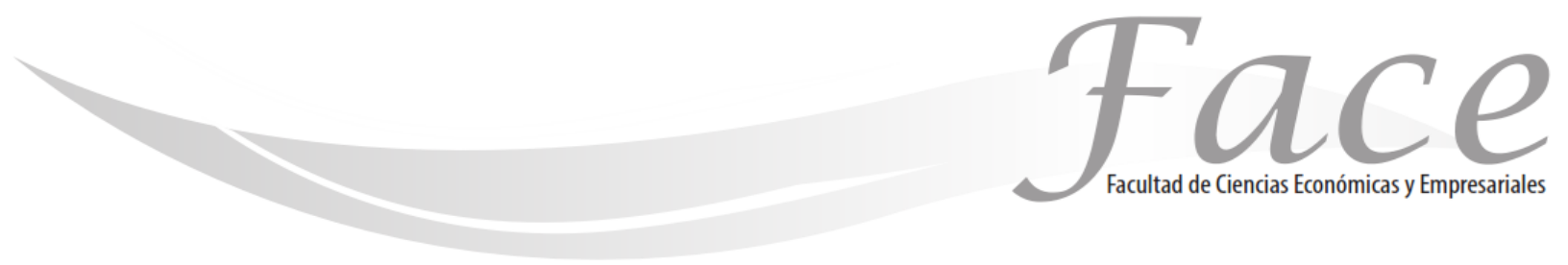

ISSN Impreso: 1794-9920

ISSN Electrónico: 2500-9338

Volumen 17-N¹

Año 2017

Págs. 65 - 72

\title{
METODOLOGIA CRM ORIENTADA A LAS UNIVERSIDADES PÚBLICAS EN COLOMBIA
}

\author{
Magda Lorena Pineda Rodríguez ${ }^{*}$ \\ Enlace ORCID: https://orcid.org/0000-0001-8139-0087 \\ John Alexander Bohada Jaime** \\ Enlace ORCID: http://orcid.org/0000-0002-3382-0190
}

Fecha de Recepción: 2 Enero 2017

Fecha de Aprobación: 8 Abril 2017

\section{Resumen:}

Este artículo, presenta una investigación acerca del diseño de una metodología para implantarla en una herramienta CRM, a fin de captar y fidelizar a los estudiantes de las universidades públicas. Para lo anterior, se realizó un trabajo de campo, diseñando un instrumento de apoyo, para percibir la razón por la cual se ha incrementado la deserción estudiantil. Los resultados arrojados, apuntaron que el servicio al cliente prestado por los empleados de la Universidad, sería un factor clave en ello. En el estudio realizado, se encontraron diversos modelos y metodologías enfocadas al servicio al cliente, viendo a éste como eje clave y fundamental de toda organización; sin embargo, todo se enfocaba en empresas comerciales. En Colombia, cuatro universidades han realizado estas implementaciones: la Universidad de Antioquia (Antioquia), la Universidad Tadeo Lozano (Bogotá), la Universidad del Norte (Barranquilla) y la Universidad de Santander (Santander), sin embargo, estas implementaciones han sido netamente administrativas. Se propone entonces realizar una implantación enfocada a procesos de captación y fidelización de estudiantes. En el diseño de la metodología, se define al estudiante como "Cliente", y a la Universidad como "Empresa", para hacer el comparativo de CRM. Se usó la metodología Exploratoria y descriptiva, y el análisis fue cuantitativo. Se diseñó una metodología para su implantación en una herramienta CRM.

Palabras Claves: CRM, Fidelización, Clientes, Deserción Estudiantil, Metodología.

\footnotetext{
* Magda Lorena Pineda Rodríguez, Magister en Dirección Estratégica con énfasis en TI, mlpineda@jdc.edu.co

** John A Bohada, Ph.D en Ingeniería del Software, jbohada@jdc.edu.co
} 


\title{
CRM METHODOLOGY ORIENTED TO PUBLIC UNIVERSITIES IN COLOMBIA
}

\begin{abstract}
This article presents an investigation about the design of a methodology to be implemented in a CRM tool, in order to attract and retain students from public universities. For the above, a field work was done, designing a support instrument, to perceive the reason for which the student's desertion has increased. The results obtained, noted that the customer service provided by employees of the University, would be a key factor in this. In the study, different models and methodologies were found and they focus on customer service, seeing it as a key and fundamental axis of any organization; however, everything was focused on commercial companies. In Colombia, four universities have made these implementations: the University of Antioquia (Antioquia), the University Tadeo Lozano (Bogotá), the University of North (Barranquilla) and the University of Santander (Santander), nevertheless, these implementations have been clearly administrative. It is proposed then to perform implantation processes focused on recruitment and retention of students. In the design of the methodology, the student is defined as "Client", and the University as "Company", to make the comparison of CRM. Exploratory and descriptive methodology was used, and the analysis was quantitative. A methodology was designed for its implementation in a CRM tool.
\end{abstract}

Keywords: CRM, Loyalty, Customers, Student Desertion, Methodology.

\section{METODOLOGIA DE CRM ORIENTADA A UNIVERSIDADES PÚBLICAS NA COLÔMBIA}

\section{Resumo}

Este artigo apresenta uma investigação sobre o desenho de uma metodologia a ser implementada em uma ferramenta de CRM, a fim de atrair e reter alunos de universidades públicas. Pelo exposto, um trabalho de campo foi feito, projetando um instrumento de apoio, para perceber a razão pela qual a deserção do estudante aumentou. Os resultados obtidos mostraram que o serviço ao cliente prestado por funcionários da Universidade, seria um fator-chave neste. No estudo, foram encontrados diferentes modelos e metodologias que se concentram no serviço ao cliente, vendo-o como um eixo fundamental de qualquer organização; No entanto, tudo estava focado em empresas comerciais. Na Colômbia, quatro universidades fizeram essas implementações: a Universidade de Antioquia (Antioquia), a Universidade Tadeo Lozano (Bogotá), a Universidade do Norte (Barranquilla) ea Universidade de Santander (Santander), no entanto, essas implementações foram claramente administrativas. Propõe-se então a realização de processos de implantação focados no recrutamento e retenção de alunos. Na concepção da metodologia, o aluno é definido como "Cliente", ea Universidade como "Empresa", para fazer a comparação do CRM. Foi utilizada metodologia exploratória e descritiva, ea análise foi quantitativa. Uma metodologia foi projetada para sua implementação em uma ferramenta de CRM.

Palavras-chave: CRM, Lealdade, Clientes, Deserção Estudantil, Metodologia. 


\section{INTRODUCCIÓN:}

La administración de la relación con los clientes, Customer Relationship Management o CRM, es un proceso iterativo, donde la información sobre el cliente se ve trasformada en relaciones provechosas. CRM como filosofía de negocio, integra distintas áreas de la empresa: ventas, marketing y servicios, las cuales al estar directamente relacionadas con el Cliente; tienen como objetivo incorporar valor añadido y crear un modelo de negocio beneficioso para ambas partes.

CRM es una estrategia no solo para conservar clientes, sino para mejorar la forma de trabajo de los empleados, consiguiendo así un mejor desempeño dentro de la empresa. CRM es la solución a los problemas de administración en la relación con los clientes, impulso ventas y esfuerzo para proyectos de marketing efectivos.

La implementación de un CRM, permite obtener mayores ingresos, vender lo máximo en las mejores condiciones, obtener nuevos clientes, fidelizar a los existentes y mantenerlos siempre satisfechos. El CRM, es un almacén de datos sobre el comportamiento de los consumidores, una estrategia de Marketing.

En Colombia, la estrategia de CRM crece a pasos agigantados, la demanda y las múltiples opciones de compra hacen que el mercado incluya estrategias que consoliden liderazgo entre empresas, y que los presupuestos de mercadeo incluyan estrategias para mantener su equilibrio. (Gamba, 2014)

Desde hace algún tiempo, la Universidad de Antioquia, ha implementado un CRM, atendiendo que los servicios que apuntan a diferentes ejes de los estudiantes (económicos, académicos, sociales, físicos y mentales), son promulgados de manera masiva, y que por ende, no siempre la información llega a los receptores interesados. Por esta razón, se asume que la implementación de esta herramienta en las Universidades, beneficia a ambas partes. Por su parte, la Universidad Tadeo Lozano, de la Ciudad de Bogotá, ha llevado a cabo la implementación de un CRM en 4 dependencias y 72 trámites. Las dependencias impactadas con el nuevo servicio son: Dirección de Gestión humana, oficina de atención a estudiantes, la secretaría general y la dirección administrativa. Ésta implementación tuvo un gran impacto en el personal de la Universidad, pues tuvieron que asistir a capacitaciones para entender la magnitud del cambio. Así mismo, la Universidad del Norte, ubicada en Barranquilla, realizo la integración de una solución de CRM con el sistema académico-administrativo, con el fin de apoyar la actualización de una única base de datos, soportar acciones de promoción, segmentación de mercados y seguimiento de las necesidades de los estudiantes. Finalmente, la Universidad de Santander, con sedes en Bogotá, Cúcuta y Valledupar, en su oficina de mercadeo institucional ha implementado un CRM, en el cual se puede consultar información sobre programas académicos, información sobre aspirantes, controlar eventos y visitas por usuario, historial de seguimiento por aspirante y generación de informes.

Hasta el momento de realizar esta investigación, en ciudades intermedias capitales de departamento, no existe ninguna Universidad con este tipo de Estrategia de Mercadeo. Las Universidades son importantes motores de investigación, innovación y plataformas de interacción social. Constituyen la base para el progreso económico y técnico en la sociedad. Se pretende entonces diseñar una metodología que pueda ser implantada en una herramienta CRM, enfocada a procesos de captación y fidelización de estudiantes, desarrollando su fase de análisis y definición de la implementación.

Diseñar una metodología que se acople a las necesidades de una universidad resulta ser innovador, ya que no existe la implantación de un CRM en instituciones de educación superior enfocadas a captar y fidelizar al cliente (estudiante).

El ajuste de las metodologías CRM al ámbito educativo, implica la capacitación y concientización del personal inmerso en el procedimiento, retroalimentaciones, análisis de amenazas y debilidades en todas las fases del proceso. Dentro de la implantación de un CRM en el ámbito educativo, diseñar una metodología que se adapte a las necesidades, resulta ser un aporte significativo, teniendo en cuenta que ésta puede ser utilizada en diversas instituciones de educación. La mayoría de planteamientos de enseñanza, tienen como referente la optimización del beneficio por cantidad.

Surge así la necesidad de incrementar los resultados de las diferentes instituciones a través de estrategias centradas en el alumno y la relación establecida con él. La fidelización del alumno, cobra un papel importante en la estrategia de toda institución, ocupando un lugar preferente ante cualquier otra. El resultado de esta investigación, podrá convertirse en un modelo a seguir por diferentes Universidades.

\section{METODOLOGÍA:}

\subsection{Materiales y Métodos}




\section{Localización}

Para efectos de realizar la investigación, se tomó como objeto de estudio la Escuela de Ciencias Tecnológicas (ECT) de la Facultad de Estudios a Distancia (FESAD), de la Universidad Pedagógica y Tecnológica de Colombia (UPTC). La población de este lugar, está dada por estudiantes, docentes y administrativos. Sin embargo, para la investigación, la población estudio será dividida en clientes internos y externos, siendo los empleados (docentes y administrativos) clientes internos y los estudiantes, clientes externos.

\section{Definición del tipo de investigación}

Según el método científico de Roberto Hernández, Carlos Fernández y Pilar Baptista (Hernández, 2014), existen dos tipos de investigación: descriptiva y exploratoria. En este caso, se aplicarán ambos tipos de investigación: la exploratoria, la cual determinará tendencias, áreas y contextos, y la descriptiva, en la que se pretende comprender el comportamiento y las características de un grupo poblacional a estudiar.

Inicialmente, se realiza la investigación exploratoria, para identificar la percepción real que se tiene, en cuanto a satisfacción del estudiante en la universidad. Luego, la descriptiva, con el fin de conocer el comportamiento de clientes (externos e internos) frente a los servicios ofrecidos por la Universidad. El análisis de la investigación, será cuantitativo.

Para Hernández, Fernández y Baptista (Hernández, 2014), la hipótesis se genera como una explicación tentativa de la investigación, y se debe formular como proposición. Una metodología acoplada a las necesidades de una organización, y su implantación en una herramienta CRM, atrae numerosos beneficios, entre ellos la captación y fidelización de los clientes.

\section{Modelos y metodologías}

Se investigaron 6 tipos de modelos CRM que podían cumplir con las necesidades: Modelo típico de un CRM, Modelo de dimensiones, Modelo de simple flujo del proceso de CRM, Modelo de Ciclo de Construcción de Relaciones, Modelo de Telesoft y Modelo estratégico CRM de Mind de Colombia. Cada uno de los modelos investigados, proponía una serie de fases que relacionan las empresas con los consumidores; así como unos ciclos donde se percibían los cambios en las estrategias empresariales.

La ardua elección del modelo, se llevó a cabo teniendo como referencia el tipo de usuarios que se manejarán: Empleados y Estudiantes, por tal motivo, se selecciona el modelo de dimensiones, el cual se forma a partir de la integración de Personas, Procesos y Tecnología. Al integrar dichas dimensiones, se puede comprender el comportamiento de los clientes, así como identificar el mercado en el cual realizar la propuesta de acuerdo a las necesidades

Tomando como base el modelo escogido, se realizó un estudio de diferentes metodologías que permitiesen la implantación de dicho modelo. Las metodologías estudiadas corresponden a: Metodología de implantación propuesta por Mind (Mind, 2015), Metodología de implantación propuesta por AELIS (AELIS, 2012) y la Metodología de implantación propuesta por García Valiente y Asoc. (Valiente, 2013). La metodología de implantación propuesta por Mind, contempla las etapas de Análisis y diseño estratégico, Planeación e implementación de procesos y recursos humanos, Planeación e implementación de datos y herramientas y Optimización modelo de negocios. Por su parte, la metodología de implantación propuesta por AELIS, estima: Planificación, Configuración y personalización, Desarrollo del Proyecto y por último, puesta en marcha. La metodología presentada por García Valiente y Asoc, contempla una serie de etapas: Definición de objetivos y visión del proyecto, Definición de la estrategia CRM, Cambios en los procesos y en el personal, Información que brindará el sistema, Tecnología que se usará, Normalización de los datos a integrar, Seguimiento, control y mejora. Ésta última metodología, permite establecer una serie de objetivos a corto plazo, los cuales, permitirán conllevar a buen término la investigación.

Teniendo en cuenta en análisis hecho a dichas metodologías de implementación, se tomó como base para esta investigación la metodología de García Valiente y Asoc. (Valiente, 2013), dado que dicha metodología en todas sus etapas, se ajustan a las necesidades del lugar objeto de esta investigación.

\section{Selección del diseño de investigación}

La investigación, incluirá un diseño no experimental, tal como Hernández, Fernández y Baptista ${ }^{1}$ lo proponen: "Es la que se realiza sin manipular deliberadamente variables". A su vez, el diseño no experimental, tiene 2 categorías: Transaccional y Longitudinal. Para este caso, se aplicará el transaccional, ya que permitirá analizar el estado de las variables en un momento dado.

\section{Muestra}

\footnotetext{
${ }^{1}$ Hernández Sampiere, R., Fernández Collado, C. Baptista Lucio, P., (2014) Metodología de la Investigación (6ta Ed). México: McGraw-Hill
} 
El tipo de muestra elegido, es el muestreo aleatorio simple (MAS), el cual permite que cada una de las personas de la población tenga la misma posibilidad de ser elegida. Teniendo en cuenta que la población es de 113 personas, de las cuales 70 son estudiantes activos y 43 son empleados activos de la universidad objeto de estudio, el tamaño de la muestra fue fijado según la siguiente expresión:

$$
n=\frac{N^{*} Z_{a}^{2} p^{*} q}{d^{2 *}(N-1)+Z_{a}^{2} p^{*} q}
$$

Donde "N" es el tamaño de la población, "Za" es el nivel de confianza adoptado (95\% para este caso), "p" proporción esperada (5\%), "q" es igual a $1-p$, (0.95 para este caso) y "d" precisión (5\% para este caso).

\subsection{Desarrollo de la Investigación}

Para la implantación del modelo de dimensiones CRM, se basó en dos fases: la fase de análisis y la fase de definición. En la fase de análisis se analizaron las necesidades de los clientes (clientes externos y clientes internos). Para tal efecto, en primera medida se utilizó la metodología de García Valiente y Asoc. (Valiente, 2013), la cual permitió definir cada uno de los elementos que la conforman y que son explicados en la tabla 1.

Tabla 1. Etapas de la Metodología García Valiente y Asoc., y su propuesta de implantación.

\begin{tabular}{|l|l|}
\hline Etapas & Propuesta \\
\hline $\begin{array}{l}\text { Definición de } \\
\text { objetivos y } \\
\text { visión del } \\
\text { proyecto }\end{array}$ & $\begin{array}{l}\text { Se planteó Misión, Visión y objetivos del } \\
\text { proyecto }\end{array}$ \\
\hline $\begin{array}{l}\text { Definición de la } \\
\text { estrategia CRM }\end{array}$ & $\begin{array}{l}\text { Capacitación de personal } \\
- \text { Marketing de programas (divulgación en } \\
\text { colegios y empresas) } \\
\text { - Modificación de mallas curriculares } \\
-\begin{array}{l}\text { Profesionalización de las carreras } \\
\text { (análisis ciclos propedéuticos) }\end{array} \\
-\begin{array}{l}\text { Mejoramiento de la calidad docente } \\
\text { (Capacitaciones) }\end{array} \\
\text { - Divulgación de servicios (Becas) } \\
\text { - Incentivos para empleados } \\
\text { - Consolidación de datos y seguimiento a } \\
\text { egresados }\end{array}$ \\
\hline $\begin{array}{l}\text { Cambios en los } \\
\text { procesos y en } \\
\text { el personal }\end{array}$ & $\begin{array}{l}\text { Se debe enfocar a los empleados hacia la } \\
\text { atención al cliente, por medio de } \\
\text { capacitaciones externas o internas, con las } \\
\text { cuales se fundamente al cliente externo } \\
\text { como eje central de todo el proceso. }\end{array}$ \\
\hline $\begin{array}{l}\text { La información que brindara el sistema, no } \\
\text { será redundante, existirá normalización de } \\
\text { que brindará el } \\
\text { sistema }\end{array}$ & \begin{tabular}{l} 
la base de datos y se podrá acceder a \\
\hline
\end{tabular} \\
\hline
\end{tabular}

\begin{tabular}{|c|c|}
\hline & 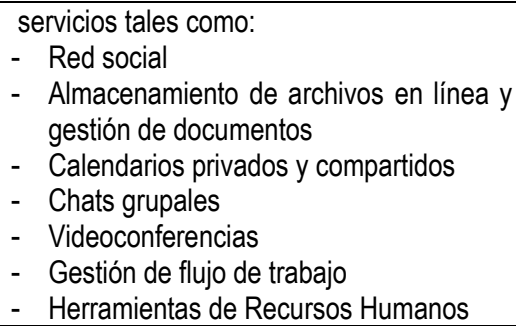 \\
\hline $\begin{array}{l}\text { Tecnología que } \\
\text { se usará }\end{array}$ & $\begin{array}{l}\text { Se recomienda Microsoft Dynamics para } \\
\text { realizar la implementación final del CRM, } \\
\text { teniendo en cuenta que ésta herramienta } \\
\text { cuenta con Integración con Outlook, } \\
\text { Reportes óptimos, Segmentación de } \\
\text { información, Mejor acceso a la información, } \\
\text { Acceso vía Web }\end{array}$ \\
\hline $\begin{array}{l}\text { Normalización } \\
\text { de los datos a } \\
\text { integrar }\end{array}$ & $\begin{array}{l}\text { Para realizar el proceso de trazabilidad de } \\
\text { los estudiantes, es necesario contar con } \\
\text { una persona que gestione la Normalización } \\
\text { en la base de datos existente, y se } \\
\text { reconsidere su diseño para que ésta pueda } \\
\text { ser compartida entre los diferentes entes } \\
\text { de la Universidad a fin de que todos } \\
\text { manejen la misma información. }\end{array}$ \\
\hline $\begin{array}{l}\text { Seguimiento, } \\
\text { control y mejora }\end{array}$ & $\begin{array}{l}\text { Es importante realizar una evaluación } \\
\text { Estudiante - Docente y una } \\
\text { autoevaluación, donde se pueda indicar el } \\
\text { cumplimiento de logros, metas y objetivos; } \\
\text { si estos se cumplieron, en qué porcentaje y } \\
\text { cuáles serían los compromisos a seguir } \\
\text { para cumplir la meta en un } 100 \% \text {. }\end{array}$ \\
\hline
\end{tabular}

En segunda medida, se definió una estructura de encuesta que permitiese detectar dichas necesidades, para los cual, se aplicaron a toda la muestra definida en secciones anteriores. La estructura general de áreas relacionadas en las encuestas es la que se describe en la tabla 2.

Tabla 2. Encuesta propuesta para cada grupo objeto de estudio

- Datos personales (Género, Edad, Programa, Semestre al que pertenece).

- Servicios de Bienestar Universitario: Permite identificar la satisfacción de los clientes con respecto a los servicios de bienestar universitario ofrecidos por la institución.

- Identidad Institucional: Permite identificar el compromiso de los clientes con la universidad.

- Gestión Administrativa: Permite identificar el compromiso percibido desde la universidad hacia los Clientes.

- Experiencia: Permite indagar a grandes rasgos las experiencias de los encuestados y su contacto con los diferentes entes de la universidad.

- Fidelización: Permite identificar la intención de recomendación de la universidad.

Basados en la estructura anterior, se definieron dos tipos de encuesta, una aplicada a los clientes internos y la otra aplicada a los clientes externos (ver anexo). Los resultados que mayor relevancia se obtuvieron para el caso de los 
clientes externos (estudiantes) fueron: el $45 \%$ desconoce los servicios prestados por la Institución, el $63 \%$ no les satisface el contacto con los empleados, el $62 \%$ percibe que el compromiso de los empleados con sus funciones es bajo, el $83 \%$ opina que las expectativas generadas al momento del ingreso a la Institución han sido cubiertas a cabalidad, el $60 \%$ están de acuerdo con los contenidos curriculares, el $79 \%$ de los encuetados considera que la divulgación de la oferta académica no es suficiente, el $91 \%$ de los estudiantes opinó que le servicio educativo contribuye a la formación de valores y finalmente, al preguntarse cuáles fueron las preferencias al momento de elegir donde formarse, el $37 \%$ respondió que la experiencia, el $33 \%$ la calidad, $27 \%$ el costo y el $3 \%$ la oportunidad.

Para el caso de los clientes internos (docentes y administrativos), los resultados que mayor relevancia tuvieron fueron los siguientes: el $42 \%$ de los empleados tienen contratación desde hace un año o menos, el $88 \%$ tienen contrataciones inferiores a un año, el $40 \%$ no está comprometido con sus funciones, el $51 \%$ opina que la motivación en el trabajo no es parte fundamental de la forma de atención de los clientes externos, el $51 \%$ no se siente satisfecho con las funciones asignadas en cumplimiento de su trabajo, el $93 \%$ aducen que no tienen un lugar de trabajo cómodo, el $33 \%$ indica que su salario no está acorde a sus funciones, el $2 \%$ indica que las relaciones con sus compañeros de trabajo no es buena, y finalmente, el $28 \%$ indica que sus funciones no están acordes con su nivel de formación.

Teniendo en cuenta los resultados de las encuestas, en la fase de definición se hizo alusión a las preguntas que se va hacer? y cuáles son los procesos a mejorar?. En este sentido, se planteó tres estrategias a seguir, la primera de ellas y en relación a que se va hacer, se sugirió realiza capacitaciones a todo los clientes internos en todo lo relacionado al servicio al cliente. En segundo lugar, para dar solución a la segunda pregunta, se sugirió mejorar los procesos relacionados a gestión y registro académico, seguimiento a egresados y atención a los servicios de los clientes externos. En este sentido, se hicieron las siguientes recomendaciones:

- Restructurar de manera gradual el plan estratégico organizacional y enfocarlo en la satisfacción del cliente.

- Restructurar de manera gradual el plan estratégico organizacional y enfocarlo en la satisfacción del cliente.

- Capacitar a empleados para los nuevos cambios estratégicos de la organización.

- Concientizar a los estudiantes y hacerlos sentir como la parte fundamental de la Universidad.
- Realizar investigaciones que conduzcan a proyectar un plan que permita divulgar los servicios ofrecidos por la universidad.

- ofrecer beneficios para los estudiantes e incentivos para los empleados.

- Importante que los lideres o jefes de oficina se deban comprometer a incentivar a sus empleados para bridar un buen servicio al estudiante.

\section{DISCUSIÓN:}

Luego de realizar el análisis de cada una de las metodologías y los modelos que pueden regir una arquitectura CRM, se analiza de manera rigurosa cómo una universidad pública debe cambiar, para satisfacer las necesidades no solo de sus clientes externos, sino de sus clientes internos. Para esto, se propone un modelo que tiene la mayoría de aspectos mencionados, además de otros, que servirán de base para el diseño de la metodología que se acople a sus necesidades.

Se debe considerar como eje central de todo proceso el cliente externo, los resultados mostrados anteriormente, dan a entender que, generalmente, en las universidades públicas, esta situación no es así, por tanto, todas las recomendaciones que se hacen, están orientadas a mejorar este aspecto. De igual forma, no se debe dejar de lado a los clientes internos, ellos son quienes a fin de cuentas harán que la propuesta realizada en este artículo, tenga continuidad, como se evidenció en las encuestas, hay que hacer un trabajo bastante grande con ellos, y parte de todo el proceso de capacitación propuesto está orientado exclusivamente a que mejoren la atención al cliente.

Los procesos por su parte, cumplen la función de articular, interconectar y compartir información con respecto a la atención de los clientes externos. Los procesos deben girar siempre en torno a ellos, tal y como se mencionó anteriormente, la tecnología cumple el papel de apoyo, teniendo en cuenta que será el "lugar" donde se puede tener una vista total de los movimientos, requerimientos y solicitudes de dichos clientes. Con la tecnología, se puede realizar la trazabilidad desde su ingreso, su permanencia y, se puede rastrear su vida laboral cuando terminen sus estudios.

\section{CONCLUSIONES:}


La deserción estudiantil, es un problema del diario vivir, que está inmerso como primera opción de todos los estudiantes ante cualquier inconveniente que se les presente, ya sea académico, administrativo o personal.

Con esta investigación, se logra obtener una visión más panorámica acerca de la percepción que tienen los estudiantes de su universidad y los servicios que presta, lo cual permite plantear una estrategia enfocada en el servicio al cliente y en la promoción y divulgación de servicios al cliente externo.

Aunque la Universidad es de carácter público, el diseño de esta metodología enfocada hacia la academia, permite rentabilidad y sostenibilidad con servicios innovadores, teniendo cliente externo como eje central, sin perder su razón de ser.

Los resultados de la investigación arrojan la importancia de manejar no solo un alto nivel académico ni acreditaciones de alta calidad, si no también, lo relevante que se ha vuelto mantener y fidelizar a los clientes.

\section{REFERENCIAS:}

Castro, A. C. (2010). Integración comercial con Microsoft Dynamics CRM en la Universidad de Montevideo. Disponible en: http://download.microsoft.com/download/D/7/4/D7 4143EE-6CD2-4FA0-B346OBACF114EDDC/Universidad\%20Montevideo, $\% 2$ OCRM, \%20Uruguay.pdf Accesado en: 18/10/2015

CRM: Administración de la Relación con los Clientes. (2014). Video Disponible en: http://campus2.funiber.org/mod/data/view.php?d= 123\&rid=7693\&filter=1 Accesado en: 18/10/2015

CRM para Educación. Proactividad, automatización y control de todo el ciclo de vida de un alumno. (2015). Disponible en: http://www.encamina.com/crm-para-educacion/ Accesado en: 28/09/2015

Díaz, F. J., Osorio, M.A y otros. (2013). Aplicando estrategias y tecnologías de Inteligencia de Negocio en sistemas de gestión académica. Accesado en: 03/10/2015

En Foco: CRM para educación. (2013) Disponible en: http://www.evaluandocrm.com/nota-3305-EnFoco-CRM-para-educacion.html Accesado en: $11 / 10 / 2015$
Evaluando CRM. Tipos de CRM. (2015). Disponible en: http://www.evaluandocrm.com/nota-4277-Tiposde-CRM.html Accesado en: 18/10/2015

Fuentes, B. O. (2014). CRM- Administración de la relación con los clientes. Disponible en: http://benchys1993.blogspot.com.co/2014/09/ane xo-de-la-guia-de-aprendizaje-n-2.html Accesado en: $15 / 10 / 2015$

GAMBA, RODRIGO. 2014. El impacto del CRM en Colombia. Disponible en http://www.dinero.com/empresas/articulo/crmcomo-estrategia-para-ganar-clientes/190729 Accesado en: 20/10/2015

Hernández Sampiere, R., Fernández Collado, C. Baptista Lucio, P., (2014) Metodología de la Investigación (6ta Ed). México: McGraw-Hill

Khodakarami, F., \& Chan, Y. E. (2014). Exploring the role of customer relationship management (CRM) systems in customer knowledge creation. Information \& Management, 51(1), 27-42. Disponible en: http://madsg.com/wpcontent/uploads/2014/01/Exploring_the_role_of_c ustomer-7339.pdf Accesado en: 27/09/2015

llumno: la primera red educativa, a nivel mundial, que logra integrar un mismo CRM. (2015). Disponible en: http://www.redilumno.com/blog/392/ilumno-laprimera-red-educativa-nivel-mundial-que-lograintegrar-un-mismo-crm Accesado en: 20/10/2015

Martínez, G. N. (2012). Mejora de la eficiencia de las PYMEs mediante la implantación de una herramienta e- CRM. Caso de Estudio: España. (Tesis) Funiber. Disponible en: http://campus2.funiber.org/mod/data/view.php?d= 123\&rid=2948\&filter=1 Accesado en: 01/10/2015

Martínez, V. (2010). Implantación CRM en un club deportivo. Funiber. Disponible en: http://campus2.funiber.org/mod/data/view.php?d= 123\&rid=12220\&filter=1 Accesado en: 18/10/2015

Ministerio de Educación de Colombia. (2013). Memorias de Encuentros Regionales de Permanencia y Graduación en Educación Superior II. Disponible en: http://www.mineducacion.gov.co/1621/w3article-315150.html Accesado en: 13/10/2015

Ministerio de Educación de Colombia. (2013). Medidas preventivas para favorecer la permanencia estudiantil. Disponible en: http://www.colombiaaprende.edu.co/html/micrositi 
os/1752/w3-article-343620.html Accesado en: $13 / 10 / 2015$

Montejo Lopera, D. A. (2010). Implementación y puesta en operación de un sistema CRM en la empresa $\mathrm{ClO}$ S.A, a partir de su estrategia y sus procesos. (Tesis) Universidad EAFIT, Medellín, Colombia. Disponible en: https://repository.eafit.edu.co/bitstream/handle/10 784/416/DiegoAlejandro_MontejoLopera_2010.pd f;jsessionid=618A0A854ACD14A8190FD8278330 3E80? sequence=1 Accesado en: 23/09/2015

Perna, A. Baraldi, E. (2014). CRM Systems in Industrial Companies: Intra- and Inter-Organizational Effects. Disponible en: https://books.google.es/books?id=PnaEAwAAQB AJ\&dq=CRM\&hl=es\&source=gbs_navlinks_s Accesado en: 15/09/2015

Pertuz, P. V. P. (2013). Estrategias para la implementación exitosa del sistema CRM en Mipymes: Planeación para transformar la relación con el cliente basado en la investigación de las Mipymes de Valledupar, Colombia. Disponible en: https://books.google.es/books?id=nj_ElwEACAAJ $\& d q=C R M \& h l=e s \& s a=X \& e i=80-F V f n G A 8 v 1$ QG2k40oBQ\&ved=0CEcQ6AEwAg Accesado en: $19 / 10 / 2015$

Petrella, C. Gestión de la relación de las universidades con docentes, estudiantes y egresados. Revista Iberoamericana de educación. Disponible en: http://www.rieoei.org/deloslectores/2470Petrella.p df Accesado en: 12/10/2015

Randall, C. (2015). Give the People What They Want: Customer Choice, Loyalty and Retention. Disponible en: http://customerthink.com/give-thepeople-what-they-want-customer-choice-loyaltyand-retention/ Accesado en: 15/10/2015

Romero Maldonado, G. (2014). Plan de marketing relacional para fidelizar a los clientes de la Escuela Rusa de Ballet en el cantón Samborondón (Doctoral dissertation). Accesado en: $21 / 10 / 2015$

SB, P., \& Bala, E. S. (2014). Role of Information Technology in Effective Implementation of Customer Relationship Management. Journal of Marketing \& Communication, 9(3). Accesado en: 21/10/2015

Urbanskienè, R., Žostautienè, D., \& Chreptavičienè, V. (2015). The model of creation of customer relationship management (CRM) system. Engineering Economics, 58(3). Accesado en: 21/10/2015 